\title{
Two new characterizations of universal integrals on the scale $[0,1]$
}

\author{
Salvatore Greco ${ }^{\mathrm{a}, \mathrm{b}}$, Radko Mesiar ${ }^{\mathrm{c}, \mathrm{d}}$, Fabio Rindone ${ }^{\mathrm{a}}$ \\ ${ }^{a}$ Department of Economics and Business 95029 Catania, Italy \\ ${ }^{b}$ Portsmouth Business School, Operations \& Systems Management University of Portsmouth \\ Portsmouth PO1 3DE, United Kingdom \\ ${ }^{c}$ Department of Mathematics and Descriptive Geometry, Faculty of Civil Engineering, Slovak \\ University of Technology, Bratislava, Slovakia \\ ${ }^{d}$ Institute of Theory of Information and Automation, Czech Academy of Sciences, Prague, Czech \\ Republic
}

\begin{abstract}
The concept of universal integral, recently proposed and axiomatized, encompasses several integrals, including the Choquet, Shilkret and Sugeno integrals. In this paper we present two new axiomatizations of universal integrals on the scale $[0,1]$. In the first characterization, we look at universal integrals on the scale $[0,1]$ as families of aggregation functions $\mathcal{F}$ satisfying some desired properties. The second characterization is given in the framing in which the original definition of universal integral was provided.
\end{abstract}

Keywords: Universal integrals, non-additive integrals, aggregation functions

\section{Introduction}

Non-additive integrals are the integrals that are based on monotone (non necessarily additive) measures. In the last decades the use of non-additive integrals has become pervasive in Decision Analysis. For example in the field of multiplecriteria decision aid (MCDA)(see [6] for a survey on MCDA) the Choquet integral [4] and the Sugeno integral [24], have become useful tools to represent interaction of criteria $[8,10]$.

Email addresses: salgreco@unict.it (Salvatore Greco), radko.mesiar@stuba.sk (Radko Mesiar), frindone@unict.it (Fabio Rindone) 
Also in decision making under risk and uncertainty the Expected Utility Theory (EUT) of von Neumann and Morgenstern [27], based on (additive) Lebesgue integral, has revealed to be inadequate to explain human behavior in many situations (see e.g. [1, 5, 14, 25]). For this motivation, more general theories, called non-EUT theories have been developed (for a seminal survey we recommend [22]). Non-EUT theories are often based on non-additive integrals. For example, in decision making under risk and uncertainty, the Choquet integral has firstly received an axiomatic characterization [19] and then has been successfully applied to economic models of decision, like the Choquet Expected Utility (CEU) of Schmeidler and Gilboa [7, 20] and the Cumulative Prospect Theory of Kahneman and Tversky [26]. Very recently, the bipolar Choquet integral of Grabisch and Lebreuche [9] has been applied in order to obtain a generalization of the CPT which does not imply gain-loss separability [13].

Klement et al. have recently proposed the concept of universal integral [17]. The family of universal integrals contain several well known non-additive integrals, like the Choquet integral [4], the Sugeno integral [24] and the Shilkret integral [21]. A further generalization is represented by the family of universal integrals computed with respect to a level dependent capacity $[15,16]$. A level dependent capacity depends also on the value of the aggregated variables and can be expressed by means of a system of capacities (see [12] for further details). Again, this concept generalizes several previous definitions, like the leveldependent Choquet integral [12], the level-dependent Shilkret integral [3] and the level-dependent Sugeno integral [18].

Any kind of integrals can be seen as a family of functionals with special properties. In the framework of MCDA these functionals turn to be special aggregation functions. In this paper we present two new characterizations of universal integrals on the scale $[0,1]$. In the first characterization we start by assuming a family of aggregation functions is given. We demonstrate that when the aggregation functions in the family satisfy a set of desired properties, then the family can be seen as a universal integral. As a consequence, we elicit a second axiomatization of universal integrals on the scale $[0,1]$ in the original setting proposed in [17]. We provide also some illustrative examples.

The paper is organized as follows. In section 2 we recall the definition of universal integral, and in section 3 we concentrate on universal integrals on the scale $[0,1]$. Section 4 shows how universal integrals can be characterized in terms of families of aggregation functions satisfying a set of given properties. In section 5 we elicit a new characterization of universal integrals on the scale $[0,1]$ in the original setting. In section 6 , we present conclusions. 


\section{Universal integrals}

A measurable space $(X, \mathcal{A})$ is a nonempty set $X$ equipped with a $\sigma-$ algebra $\mathcal{A}$. Given a measurable space $(X, \mathcal{A})$, a function $f: X \rightarrow[0, \infty]$ is $\mathcal{A}$-measurable if, for each $B \in \mathcal{B}([0, \infty])$, the $\sigma$-algebra of Borel subsets of $[0, \infty]$, the preimage $f^{-1}(B)$ is an element of $\mathcal{A}$.

For each $A \subseteq X$ we denote with $\mathbf{1}_{A}$ the function on $X$ defined by: $\mathbf{1}_{A}(x)=1$ if $x \in A, \mathbf{1}_{A}(x)=0$ else.

A monotone measure on a measurable space $(X, \mathcal{A})$ is a function $m: \mathcal{A} \rightarrow[0, \infty]$ satisfying the following conditions:

1. boundary conditions: $m(\emptyset)=0$ and $m(X)>0$;

2. monotonicity: $m(A) \leq m(B)$ for all $A, B \in \mathcal{A}$ such that $A \subseteq B$.

A monotone measure $m$ satisfying $m(X)=1$ is also called capacity or fuzzy measure $[24,4]$.

Let $(X, \mathcal{A})$ be a measurable space. We shall use the following notations:

1. $\mathcal{F}^{(X, \mathcal{A})}$ denotes the set of all $\mathcal{A}$-measurable functions $f: X \rightarrow[0, \infty]$; for all $f \in \mathcal{F}^{(X, \mathcal{A})}$, the level set $\{x \in X \mid f(x) \geq t\}, t \in[0,1]$, is briefly denoted by $\{f \geq t\}$;

2. for each $a \in] 0, \infty], \mathcal{M}_{a}^{(X, \mathcal{A})}$ denotes the set of all monotone measures on $(X, \mathcal{A})$ satisfying $m(X)=a$, and we put

$$
\mathcal{M}^{(X, \mathcal{A})}=\bigcup_{a \in] 0, \infty]} \mathcal{M}_{a}^{(X, \mathcal{A})} ;
$$

3. let $\mathcal{S}$ be the class of all measurable spaces, and put

$$
\mathcal{D}_{[0, \infty]}=\bigcup_{(X, \mathcal{A}) \in \mathcal{S}} \mathcal{M}^{(X, \mathcal{A})} \times \mathcal{F}^{(X, \mathcal{A})}
$$

Definition 1 A pseudo-multiplication is a function $\otimes:[0, \infty]^{2} \rightarrow[0, \infty]$ such that for all $x, y, t$ and $z \in[0, \infty]$ the following properties are satisfied:

- monotonicity: $x \otimes y \leq t \otimes z$, whenever $x \leq t$ and $y \leq z$;

- zero is an annihilator: $x \otimes 0=0 \otimes x=0$;

- neutral element: there exists $e \in] 0, \infty]$ such that $e \otimes x=x \otimes e=x$. 
In [17] the following definition has been given.

Definition 2 A function $I: \mathcal{D}_{[0, \infty]} \rightarrow[0, \infty]$ is called a universal integral, if the following axioms hold:

(I1) for any measurable space $(X, \mathcal{A})$, the restriction of I to $\mathcal{M}^{(X, \mathcal{A})} \times \mathcal{F}^{(X, \mathcal{A})}$ is nondecreasing in each coordinate;

(I2) there exists a pseudo-multiplication $\otimes:[0, \infty]^{2} \rightarrow[0, \infty]$ such that for all $(X, \mathcal{A}) \in \mathcal{S}, m \in \mathcal{M}^{(X, \mathcal{A})}, A \in \mathcal{A}$ and $c \in[0, \infty]$,

$$
\boldsymbol{I}\left(m, c \cdot \mathbf{1}_{A}\right)=c \otimes m(A) ;
$$

(I3) $\boldsymbol{I}\left(m_{1}, f_{1}\right)=\boldsymbol{I}\left(m_{2}, f_{2}\right)$ for all $\left(m_{1}, f_{1}\right),\left(m_{2}, f_{2}\right) \in \mathcal{D}_{[0, \infty]}$ such that

$$
\left.\left.m_{1}\left(\left\{f_{1} \geq t\right\}\right)=m_{2}\left(\left\{f_{2} \geq t\right\}\right), \text { for all } t \in\right] 0, \infty\right] .
$$

For each pair $(m, f) \in \mathcal{D}_{[0, \infty]}$, consider the function $\left.\left.h^{(m, f)}:\right] 0, \infty\right] \rightarrow[0, \infty]$ defined by $h^{(m, f)}(t)=m(\{f \geq t\})$, for all $\left.\left.t \in\right] 0, \infty\right]$. For each $(m, f) \in \mathcal{D}_{[0, \infty]}$, $h^{(m, f)}$ is nonincreasing and Borel measurable. Observe that the function $\left.h^{(} m, f\right)$ can be seen as a generalized survival function (dual to the distribution function for a random variable), and then the axiom (I3) expresses a generalization of a well known fact from the probability theory, namely, that two random variables possessing the same distribution function have the same expected value. Similarly, axiom (I2) can be seen as a generalization of the fact that if a random variable $V$ has as its range $\{0, c\}$ for some constant $c$, then its expected value depends only on $c$ and $P(\{V=c\})$.

Let $\mathcal{W}$ denote the subset of all nonincreasing functions from $\mathcal{F}^{(10, \infty], \mathcal{B}(] 0, \infty]))}$. The following characterization theorem, linking an approach to fuzzy integrals introduced in [23] and universal integrals, was shown in [17].

Theorem 1 A function I : $\mathcal{D}_{[0, \infty]} \rightarrow[0, \infty]$ is a universal integral related to some pseudo-multiplication $\otimes$ if and only if there is a function $\boldsymbol{J}: \mathcal{W} \rightarrow[0, \infty]$ satisfying the following conditions:

(J1) $\boldsymbol{J}$ is nondecreasing;

(J2) $\boldsymbol{J}\left(d \cdot \mathbf{1}_{0, c]}\right)=c \otimes d$, for all $c, d \in[0, \infty]$;

(J3) $\boldsymbol{J}\left(h^{(m, f)}\right)=\boldsymbol{I}(m, f)$ for all $(m, f) \in \mathcal{D}_{[0, \infty]}$. 


\section{Universal integrals on the scale $[0,1]$}

In the previous section we have recalled the original definition of universal integrals. Nevertheless, from here on we focus on the unit interval $[0,1]$. Let $\mathcal{F}_{[0,1]}^{(X, \mathcal{A})} \subset \mathcal{F}^{(X, \mathcal{A})}$ the set of measurable functions with range in $[0,1]$. The restriction of a universal integral to

$$
\mathcal{D}_{[0,1]}=\bigcup_{(X, \mathcal{A}) \in \mathcal{S}} \mathcal{M}_{1}^{(X, \mathcal{A})} \times \mathcal{F}_{[0,1]}^{(X, \mathcal{A})},
$$

is called a universal integral on the scale $[0,1]$ (see [17, Sec. V]). Universal integrals on the scale $[0,1]$ are related to pseudo-multiplications with 1 as neutral element, called semicopulas [2].

Definition 3 A semicopula is a function $\otimes:[0,1]^{2} \rightarrow[0,1]$ such that for all $x, y, t$ and $z \in[0,1]$ the following axioms are satisfied:

- monotonicity: $x \otimes y \leq t \otimes z$, whenever $x \leq t$ and $y \leq z$;

- 1 is a neutral element: $1 \otimes x=x \otimes 1=x$.

Note that a semicopula has 0 as annihilator: it holds $0 \leq a \otimes 0 \leq 1 \otimes 0=0$ and $0 \leq 0 \otimes a \leq 0 \otimes 1=0$. A commutative and associative semicopula is called a $t$-norm. Typical examples of t-norms are the minimum $(\wedge)$, the product $(\cdot)$, and the Lukasiewicz t-norm defined by $T_{L}(x, y)=(x+y-1) \vee 0$.

By the sake of clarity, we rewrite definition 2 of universal integrals adapted to the scale $[0,1]$.

Definition 4 [17] A function I : $\mathcal{D}_{[0,1]} \rightarrow[0,1]$ is called a universal integral on the scale $[0,1]$ if it satisfies the following axioms:

$\left(I 1^{*}\right)$ for any measurable space $(X, \mathcal{A})$, the restriction of $\mathbf{I}$ to $\mathcal{M}_{1}^{(X, \mathcal{A})} \times \mathcal{F}_{[0,1]}^{(X, \mathcal{A})}$ is nondecreasing in each coordinate;

$(I 2 *)$ there exists a semicopula $\otimes:[0,1]^{2} \rightarrow[0,1]$ such that for all $(X, \mathcal{A}) \in \mathcal{S}$, $m \in \mathcal{M}_{1}^{(X, \mathcal{A})}, A \in \mathcal{A}$ and $c \in[0,1]$,

$$
\boldsymbol{I}\left(m, c \cdot \mathbf{1}_{A}\right)=c \otimes m(A)
$$

$\left(I^{*}\right) \boldsymbol{I}\left(m_{1}, f_{1}\right)=\boldsymbol{I}\left(m_{2}, f_{2}\right)$ for all $\left(m_{1}, f_{1}\right),\left(m_{2}, f_{2}\right) \in \mathcal{D}_{[0,1]}$ such that

$$
\left.\left.m_{1}\left(\left\{f_{1} \geq t\right\}\right)=m_{2}\left(\left\{f_{2} \geq t\right\}\right) \text { for all } t \in\right] 0,1\right] \text {. }
$$




\subsection{Universal integrals on the scale $[0,1]$ as aggregation operators}

Let $(X, \mathcal{A})$ be a measurable space. For any pair of measurable functions $f, g$ from $\mathcal{F}_{[0,1]}^{(X, \mathcal{A})}$, we say that $f$ is greater than $g$ and we write $g \leq f$ if $g(x) \leq f(x)$ for all $x \in X$. Obviously, with respect to $\leq$ the set $\mathcal{F}_{[0,1]}^{(X, \mathcal{A})}$ is equipped with minimum and maximum, being for all $f \in \mathcal{F}_{[0,1]}^{(X, \mathcal{A})}, \mathbf{1}_{\emptyset} \leq f \leq \mathbf{1}_{X}$.

Consider a function $\mathbf{H}: \mathcal{F}_{[0,1]}^{(X, \mathcal{A})} \rightarrow[0,1]$. We say that $\mathbf{H}$ is monotone if $g \leq f$ implies $\mathbf{H}(g) \leq \mathbf{H}(f)$ for all $f, g \in \mathcal{F}_{[0,1]}^{(X, \mathcal{A})}$. A monotone function on $\mathcal{F}_{[0,1]}^{(X, \mathcal{A})}$ satisfying boundary conditions is called an aggregation function.

Definition 5 [11] A monotone function $\boldsymbol{H}: \mathcal{F}_{[0,1]}^{(X, \mathcal{A})} \rightarrow[0,1]$ such that

- $\boldsymbol{H}\left(\mathbf{1}_{\emptyset}\right)=0$, and

- $\boldsymbol{H}\left(\mathbf{1}_{X}\right)=1$,

is called a $[0,1]$-valued aggregation function on $(X, \mathcal{A})$.

We denote by $\mathcal{H}_{[0,1]}^{(X, \mathcal{A})}$ the set of all $[0,1]$-valued aggregation functions on $(X, \mathcal{A})$ and we put

$$
\mathcal{H}_{[0,1]}=\bigcup_{(X, \mathcal{A}) \in \mathcal{S}} \mathcal{H}_{[0,1]}^{(X, \mathcal{A})}
$$

In the sequel we shall denote $\mathcal{H}_{[0,1]}^{(X, \mathcal{A})}$ with $\mathcal{H}^{(X, \mathcal{A})}$.

Remark 1 For each $(X, \mathcal{A}) \in \mathcal{S}$ and $\boldsymbol{H} \in \mathcal{H}^{(X, \mathcal{A})}$, the function $m_{\boldsymbol{H}}: \mathcal{A} \rightarrow[0,1]$ defined by

$$
m_{\boldsymbol{H}}(E)=\boldsymbol{H}\left(\mathbf{1}_{E}\right) \text { for all } E \in \mathcal{A}
$$

is a capacity of $\mathcal{M}_{1}^{(X, \mathcal{A})}$. Indeed, $m_{\boldsymbol{H}}(\emptyset)=\boldsymbol{H}\left(\mathbf{1}_{\emptyset}\right)=0$ and $m_{\boldsymbol{H}}(X)=\boldsymbol{H}\left(\mathbf{1}_{X}\right)=1$ by definition of aggregation function. Moreover, for all $E, F \in \mathcal{A}$ with $E \subseteq F$, $m_{\boldsymbol{H}}(E)=\boldsymbol{H}\left(\mathbf{1}_{E}\right) \leq \boldsymbol{H}\left(\mathbf{1}_{F}\right)=m_{\boldsymbol{H}}(F)$ by monotonicity of $\boldsymbol{H}$. We call $m_{\boldsymbol{H}}$ the capacity induced by $\boldsymbol{H}$ and, obviously, two different aggregation functions can induce the same capacity, provided that they coincide on characteristic functions.

Suppose $\mathbf{I}: \mathcal{D}_{[0,1]} \rightarrow[0,1]$ is a universal integral on $[0,1]$ related to some semicopula $\otimes$. For all $(X, \mathcal{A}) \in \mathcal{S}$ and $m \in \mathcal{M}_{1}^{(X, \mathcal{A})}$ the function $\mathbf{I}_{m}: \mathcal{F}_{[0,1]}^{(X, \mathcal{A})} \rightarrow[0,1]$ defined by $\mathbf{I}_{m}(f)=\mathbf{I}(m, f)$, for all $f \in \mathcal{F}_{[0,1]}^{(X, \mathcal{A})}$, is an aggregation function of $\mathcal{H}^{(X, \mathcal{A})}$. Indeed, if $f, g \in \mathcal{F}_{[0,1]}^{(X, \mathcal{A})}$ with $g \leq f$, then $\mathbf{I}_{m}(g)=\mathbf{I}(m, g) \leq$ 
$\mathbf{I}_{m}(f)=\mathbf{I}(m, f)$ by monotonicity of $\mathbf{I}$; moreover $\mathbf{I}_{m}\left(\mathbf{1}_{\emptyset}\right)=\mathbf{I}\left(m, \mathbf{1}_{\emptyset}\right)=0$ and $\mathbf{I}_{m}\left(\mathbf{1}_{X}\right)=\mathbf{I}\left(m, \mathbf{1}_{X}\right)=1$ by idempotency of $\mathbf{I}$. Thus, any universal integral on the scale $[0,1]$ can be viewed as a family of aggregation functions, one for each

$m \in \bigcup_{(X, \mathcal{A}) \in \mathcal{S}} \mathcal{M}_{1}^{(X, \mathcal{A})}$. In the next section we shall characterize universal integrals on the scale $[0,1]$, starting from a family of aggregation functions of $\mathcal{H}_{[0,1]}$ satisfying some given properties.

\section{Axiomatic foundation of universal integrals on the scale $[0,1]$ in terms of aggregation functions}

Let us consider a family $\mathcal{F} \subseteq \mathcal{H}_{[0,1]}$ with $\mathcal{F} \neq \emptyset$ and consider the following axioms:

(A1) For all $\mathbf{H}, \mathbf{G} \in \mathcal{F}$ such that $\mathbf{H} \in \mathcal{H}^{\left(X_{1}, \mathcal{A}_{1}\right)}$ and $\mathbf{G} \in \mathcal{H}^{\left(X_{2}, \mathcal{A}_{2}\right)}$ and for all $h \in \mathcal{F}_{[0,1]}^{\left(X_{1}, \mathcal{A}_{1}\right)}$ and $g \in \mathcal{F}_{[0,1]}^{\left(X_{2}, \mathcal{A}_{2}\right)}$ such that $\mathbf{H}\left(\mathbf{1}_{\{h \geq t\}}\right) \geq \mathbf{G}\left(\mathbf{1}_{\{g \geq t\}}\right)$ for all $t \in[0,1]$, then

$$
\mathbf{H}(h) \geq \mathbf{G}(g) .
$$

(A2) Every aggregation function in $\mathcal{F}$ is idempotent, i.e. for all $\mathbf{H} \in \mathcal{F} \cap \mathcal{H}^{(X, \mathcal{A})}$,

$$
\mathbf{H}\left(c \cdot \mathbf{1}_{X}\right)=c, \quad \text { for all } c \in[0,1] .
$$

(A3) For all $(X, \mathcal{A}) \in \mathcal{S}$ and $m \in \mathcal{M}_{1}^{(X, \mathcal{A})}$ there exists $\mathbf{H} \in \mathcal{F} \cap \mathcal{H}^{(X, \mathcal{A})}$ such that

$$
\mathbf{H}\left(\mathbf{1}_{E}\right)=m(E) \text { for all } E \in \mathcal{A} \text {. }
$$

Remark 2 Observe that axioms (A1), (A2) and (A3) are independent as it is shown by the following examples, in which two of the above axioms hold, but the remaining is not valid.

1) For all $(X, \mathcal{A}) \in \mathcal{S}$ consider the set of finite weighting functions on $X$

$$
W_{X}=\left\{\omega: X \rightarrow[0,1] \mid \#\{x \in X \mid \omega(x)>0\}<\infty \text { and } \sum_{x \in X} \omega(x)=1\right\},
$$

and the set of of all weighted averages on $\mathcal{F}_{[0,1]}^{(X, \mathcal{A})}$

$$
W A^{(X, \mathcal{A})}=\left\{\boldsymbol{H}_{\omega}: \mathcal{F}_{[0,1]}^{(X, \mathcal{A})} \rightarrow[0,1] \mid \boldsymbol{H}_{\omega}(f)=\sum_{x \in X} \omega(x) f(x), \omega \in W_{X}\right\} .
$$

If we consider the family of all weighted averages $\mathcal{F}=\bigcup_{(X, \mathcal{A})} W A^{(X, \mathcal{A})}$, this satisfies axioms (A1) and (A2) but not axiom (A3). 
2) For all $(X, \mathcal{A}) \in \mathcal{S}$ and for all $m \in M_{1}^{(X, \mathcal{A})}$ let us consider the aggregation function $\boldsymbol{H}_{m}: \mathcal{F}_{[0,1]}^{(X, \mathcal{A})} \rightarrow[0,1]$ defined for all $f \in \mathcal{F}_{[0,1]}^{(X, \mathcal{A})}$ as

$$
\boldsymbol{H}_{m}(f)=\int_{0}^{1} m(\{f \geq \sqrt{t}\}) d t .
$$

If we consider the family $\mathcal{F}=\left\{\boldsymbol{H}_{m} \mid m \in \bigcup_{(X, \mathcal{A}) \in \mathcal{S}} M_{1}^{(X, \mathcal{A})}\right\}$ this satisfies axioms (A1) and (A3) but not axiom (A2).

3) For all $(X, \mathcal{A}) \in \mathcal{S}$ and for all $m_{1}, m_{2} \in M_{1}^{(X, \mathcal{A})}$ consider the aggregation function $\boldsymbol{H}_{m_{1}, m_{2}}: \mathcal{F}_{[0,1]}^{(X, \mathcal{A})} \rightarrow[0,1]$ defined for all $f \in \mathcal{F}_{[0,1]}^{(X, \mathcal{A})}$ as

$$
\boldsymbol{H}_{m_{1}, m_{2}}(f)=\int_{0}^{0.5} m_{1}(\{f \geq t\}) d t+\int_{0.5}^{1} m_{2}(\{f \geq t\}) d t .
$$

If we consider the family $\mathcal{F}=\left\{\boldsymbol{H}_{m_{1}, m_{2}} \mid m_{1}, m_{2} \in \bigcup_{(X, \mathcal{A}) \in \mathcal{S}} M_{1}^{(X, \mathcal{A})}\right\}$, this satisfies axioms (A2) and (A3) but not axiom (A1). Indeed in this case consider $X=\{1,2\}, f: X \rightarrow[0,1]$ defined by $f(1)=0.2, f(2)=0.4$ and the capacities $m_{1}, m_{2}, m_{1}^{\prime}, m_{2}^{\prime}$ defined by $m_{1}(\{1\})=m_{1}(\{2\})=0.5$, $m_{2}(\{1\})=m_{2}(\{2\})=0, m_{1}^{\prime}(\{1\})=1, m_{1}^{\prime}(\{2\})=0$, and $m_{2}^{\prime}(\{1\})=$ $0, m_{2}^{\prime}(\{2\})=0.5$. It results that $\boldsymbol{H}_{m_{1}, m_{2}}\left(1_{\{f \geq t\}}\right)=\boldsymbol{H}_{m_{1}^{\prime}, m_{2}^{\prime}}\left(1_{\{f \geq t\}}\right)$ for all $t \in[0,1]$ but $\boldsymbol{H}_{m_{1}, m_{2}}(f)=0.3>\boldsymbol{H}_{m_{1}^{\prime}, m_{2}^{\prime}}(f)=2$, which contradicts axiom (A1).

Theorem 2 Axioms (A1), (A2) and (A3) hold for a family $\mathcal{F} \subseteq \mathcal{H}_{[0,1]}$ if and only if there exists a universal integral $\boldsymbol{I}: \mathcal{D}_{[0,1]} \rightarrow[0,1]$ with a semicopula $\otimes$ such that, for all $\boldsymbol{H} \in \mathcal{F} \cap \mathcal{H}^{(X, \mathcal{A})}$

$$
\boldsymbol{H}(h)=\boldsymbol{I}\left(m_{\boldsymbol{H}}, h\right), \quad \text { for all } h \in \mathcal{F}_{[0,1]}^{(X, \mathcal{A})} .
$$

More precisely, for all $(a, b) \in[0,1]^{2}$ we define

$$
a \otimes b=\boldsymbol{H}\left(a \cdot \mathbf{1}_{B}\right) \text { with } \boldsymbol{H} \in \mathcal{F} \cap \mathcal{H}^{(X, \mathcal{A})}, B \in \mathcal{A} \text { such that } \boldsymbol{H}\left(\mathbf{1}_{B}\right)=b .
$$

\section{Proof}

Let us start by proving that if axioms (A1)-(A3) hold for a family of aggregation functions $\mathcal{F}$, then, necessarily, there exists a universal integral satisfying 
equation (2). Let us note that any family $\mathcal{F}$ satisfying axiom (A3) is, consequently, nonempty.

We begin the proof by showing that function $\otimes$ defined in (3) is a semicopula. First we prove that definition of $\otimes$ is well posed and then that it defines a semicopula.

Observe that for all $a, b \in[0,1]$ and for all $\mathbf{H} \in \mathcal{F} \cap \mathcal{H}^{\left(X_{1}, \mathcal{A}_{1}\right)}, \mathbf{G} \in \mathcal{F} \cap \mathcal{H}^{\left(X_{2}, \mathcal{A}_{2}\right)}$ and $B \in \mathcal{A}_{1}, C \in \mathcal{A}_{2}$ such that $\mathbf{H}\left(\mathbf{1}_{B}\right)=\mathbf{G}\left(\mathbf{1}_{C}\right)=b$, then $\mathbf{H}\left(a \cdot \mathbf{1}_{B}\right)=\mathbf{G}\left(a \cdot \mathbf{1}_{C}\right)$ by (A1). Indeed

$$
\mathbf{H}\left(\mathbf{1}_{\left\{a \cdot \mathbf{1}_{B} \geq t\right\}}\right)=\mathbf{G}\left(\mathbf{1}_{\left\{a \cdot \mathbf{1}_{C} \geq t\right\}}\right)=\left\{\begin{array}{lc}
\mathbf{H}\left(\mathbf{1}_{X_{1}}\right)=\mathbf{G}\left(\mathbf{1}_{X_{2}}\right)=1 & t=0 \\
\mathbf{H}\left(\mathbf{1}_{B}\right)=\mathbf{G}\left(\mathbf{1}_{C}\right)=b & 0<t \leq a \\
\mathbf{H}\left(\mathbf{1}_{\emptyset}\right)=\mathbf{G}\left(\mathbf{1}_{\emptyset}\right)=0 & a<t \leq 1 .
\end{array}\right.
$$

Moreover, let us note that for any $b \in[0,1]$ there exist $\mathbf{H} \in \mathcal{F} \cap \mathcal{H}^{(X, \mathcal{A})}$ and $E \in \mathcal{A}$ such that $\mathbf{H}\left(\mathbf{1}_{E}\right)=b$. Indeed if $b=0$ or $b=1$, then for any $(X, \mathcal{A}) \in \mathcal{S}$ and any $\mathbf{H} \in \mathcal{F} \cap \mathcal{H}^{(X, \mathcal{A})}, \mathbf{H}\left(\mathbf{1}_{\emptyset}\right)=0$ and $\mathbf{H}\left(\mathbf{1}_{X}\right)=1$. If $0<b<1$ we consider for some $(X, \mathcal{A}) \in \mathcal{S}$ with $\# X \geq 2$ the capacity $m^{*} \in \mathcal{M}_{1}^{(X, \mathcal{A})}$ such that $m^{*}(\emptyset)=0$, $m^{*}(X)=1$ and $m^{*}(E)=b$ for all $E \in \mathcal{A} \backslash\{\emptyset, X\}$; then by axiom (A3), there exists $\mathbf{H} \in \mathcal{F} \cap \mathcal{H}^{(X, \mathcal{A})}$ such that $\mathbf{H}\left(\mathbf{1}_{E}\right)=m^{*}(E)=b$ for some $E \in \mathcal{A}$. Thus, we conclude that $\otimes$ is well defined.

Now we prove that $\otimes$ is nondecreasing. Let us take $0 \leq a_{1} \leq a_{2} \leq 1$ and $0 \leq b_{1} \leq b_{2} \leq 1$. By $\left(A_{3}\right)$ there exist $\mathbf{H} \in \mathcal{F} \cap \mathcal{H}^{\left(X_{1}, \mathcal{A}_{1}\right)}, \overline{\mathbf{G}} \in \mathcal{F} \cap \mathcal{H}^{\left(X_{2}, \mathcal{A}_{2}\right)}$ and $B \in \mathcal{A}_{1}, C \in \mathcal{A}_{2}$ such that $\mathbf{H}\left(\mathbf{1}_{B}\right)=b_{1}$ and $\mathbf{G}\left(\mathbf{1}_{C}\right)=b_{2}$. By definition, $a_{1} \otimes b_{1}=$ $\mathbf{H}\left(a_{1} \cdot \mathbf{1}_{B}\right)$ and $a_{2} \otimes b_{2}=\mathbf{G}\left(a_{2} \cdot \mathbf{1}_{C}\right)$. It results that $\mathbf{H}\left(\mathbf{1}_{\left\{a_{1} \cdot \mathbf{1}_{B} \geq t\right\}}\right) \leq \mathbf{G}\left(\mathbf{1}_{\left\{a_{2} \cdot \mathbf{1}_{B} \geq t\right\}}\right)$ for all $t \in[0,1]$, indeed

$$
\begin{array}{r}
\mathbf{H}\left(\mathbf{1}_{\left\{a_{1} \cdot \mathbf{1}_{B} \geq t\right\}}\right)=\left\{\begin{array}{lc}
\mathbf{H}\left(\mathbf{1}_{X_{1}}\right)=1 & t=0 \\
\mathbf{H}\left(\mathbf{1}_{B}\right)=b_{1} & 0<t \leq a_{1} \\
\mathbf{H}\left(\mathbf{1}_{\emptyset}\right)=0 & a_{1}<t \leq 1,
\end{array}\right. \\
\mathbf{G}\left(\mathbf{1}_{\left\{a_{2} \cdot \mathbf{1}_{C} \geq t\right\}}\right)=\left\{\begin{array}{lc}
\mathbf{H}\left(\mathbf{1}_{X_{2}}\right)=1 & t=0 \\
\mathbf{H}\left(\mathbf{1}_{C}\right)=b_{2} & 0<t \leq a_{2} \\
\mathbf{H}\left(\mathbf{1}_{\emptyset}\right)=0 & a_{2}<t \leq 1 .
\end{array}\right.
\end{array}
$$

It follows from (A1) that $a_{1} \otimes b_{1} \leq a_{2} \otimes b_{2}$ and then $\otimes$ is monotone.

Now we prove that $c \otimes 1=1 \otimes c=c$ for all $c \in[0,1]$. We have $c \otimes 1=\mathbf{H}\left(c \cdot \mathbf{1}_{X}\right)=$ $c$ for all $\mathbf{H} \in \mathcal{F} \cap \mathcal{H}^{(X, \mathcal{A})}$ by (A2). Moreover, we have $1 \otimes c=\mathbf{H}\left(1 \cdot \mathbf{1}_{C}\right)=\mathbf{H}\left(\mathbf{1}_{C}\right)$ for some $\mathbf{H} \in \mathcal{F} \cap \mathcal{H}^{(X, \mathcal{A})}$ and some $C \in \mathcal{A}$ such that $\mathbf{H}\left(\mathbf{1}_{C}\right)=c$, by definition of $\otimes$. 
Now we prove that aggregation functions from family $\mathcal{F}$ define a universal integral I : $\mathcal{D}_{[0,1]} \rightarrow[0,1]$ with respect to the semicopula $\otimes$, by means of

$$
\mathbf{I}(m, f)=\mathbf{H}_{m}(f) \quad \text { for all } \quad(m, f) \in \mathcal{D}_{[0,1]}
$$

and being

$$
\mathbf{H}_{m} \in \mathcal{H}^{(X, \mathcal{A})} \quad \text { such that } \quad \mathbf{H}_{m}\left(\mathbf{1}_{E}\right)=m(E) \text {, for all } E \in \mathcal{A} .
$$

First let us note how, by axiom (A3), for any $m \in \mathcal{M}_{1}^{(X, \mathcal{A})}$, there exist such an $\mathbf{H}_{m} \in \mathcal{H}^{(X, \mathcal{A})}$ and, moreover, by axiom (A1) it is unique, and, consequently, the function $\mathbf{I}$ is well defined.

Let us consider $(m, f),\left(m^{\prime}, f^{\prime}\right) \in \mathcal{M}_{1}^{(X, \mathcal{A})} \times \mathcal{F}_{[0,1]}^{(X, \mathcal{A})}$ such that $m \geq m^{\prime}$ and $f \geq f^{\prime}$. By definition of $\mathbf{I}$, the monotonicity condition $\mathbf{I}(m, f) \geq \mathbf{I}\left(m^{\prime}, f^{\prime}\right)$ reduces to $\mathbf{H}_{m}(f) \geq \mathbf{H}_{m^{\prime}}\left(f^{\prime}\right)$, which is true by (A1), being $\mathbf{H}_{m}\left(\mathbf{1}_{\{f \geq t\}}\right)=m(\{f \geq t\}) \geq$ $m^{\prime}\left(\left\{f^{\prime} \geq t\right\}\right)=\mathbf{H}_{m^{\prime}}\left(\mathbf{1}_{\left\{f^{\prime} \geq t\right\}}\right)$ for all $t \in[0,1]$. In this way we proved that condition $\left(\mathrm{I} 1^{*}\right)$ in definition 4 holds.

Condition (I2*) holds by definition of $\otimes$ and $\mathbf{I}$. Indeed, for all $\left(m, c \cdot \mathbf{1}_{E}\right) \in \mathcal{D}_{[0,1]}$, $\boldsymbol{I}\left(m, c \cdot \mathbf{1}_{E}\right)=\mathbf{H}_{m}\left(c \cdot \mathbf{1}_{E}\right)=c \otimes \mathbf{H}_{m}\left(\mathbf{1}_{E}\right)=c \otimes m(E)$.

Finally, if for $\left(m_{1}, f_{1}\right) \in \mathcal{M}_{1}^{\left(X_{1}, \mathcal{A}_{1}\right)} \times \mathcal{F}_{[0,1]}^{\left(X_{1}, \mathcal{A}_{1}\right)},\left(m_{2}, f_{2}\right) \in \mathcal{M}_{1}^{\left(X_{1}, \mathcal{A}_{1}\right)} \times \mathcal{F}_{[0,1]}^{\left(X_{2}, \mathcal{A}_{2}\right)}$ it results that $m_{1}\left(\left\{f_{1} \geq t\right\}\right)=m_{2}\left(\left\{f_{2} \geq t\right\}\right)$ for all $t \in[0,1]$ then we have $\mathbf{H}_{m_{1}}\left(\mathbf{1}_{\left\{f_{1} \geq t\right\}}\right)=\mathbf{H}_{m_{2}}\left(\mathbf{1}_{\left\{f_{2} \geq t\right\}}\right)$ for all $t \in[0,1]$.

Hence, by (A1) we have $\mathbf{H}_{m_{1}}\left(f_{1}\right)=\mathbf{H}_{m_{2}}\left(f_{2}\right)$, i.e. $\mathbf{I}\left(m_{1}, f_{1}\right)=\mathbf{I}\left(m_{2}, f_{2}\right)$ such that also (I3*) holds.

Now we prove the sufficiency part. Suppose that $\mathbf{I}: \mathcal{D}_{[0,1]} \rightarrow[0,1]$ is a universal integral with respect to some semicopula $\otimes$. For all $(X, \mathcal{A}) \in \mathcal{S}$ and $m \in \mathcal{M}_{1}^{(X, \mathcal{A})}$ the function $\mathbf{I}_{m}: \mathcal{F}_{[0,1]}^{(X, \mathcal{A})} \rightarrow[0,1]$ defined by $\mathbf{I}_{m}(f)=\mathbf{I}(m, f)$ for all $f \in \mathcal{F}_{[0,1]}^{(X, \mathcal{A})}$, is an aggregation function of $\mathcal{H}^{(X, \mathcal{A})}$. Let us prove that the family of aggregation functions $\mathcal{F}=\left\{\mathbf{I}_{m} \mid m \in \bigcup_{(X, \mathcal{A}) \in \mathcal{S}} \mathcal{M}_{1}^{(X, \mathcal{A})}\right\}$ satisfies axioms (A1)-(A3). Suppose that for some $m_{1} \in \mathcal{M}_{1}^{\left(X_{1}, \mathcal{A}_{1}\right)}, m_{2} \in \mathcal{M}_{1}^{\left(X_{1}, \mathcal{A}_{1}\right)}$ and $f_{1} \in \mathcal{F}_{[0,1]}^{\left(X_{1}, \mathcal{A}_{1}\right)}$ and $f_{2} \in \mathcal{F}_{[0,1]}^{\left(X_{2}, \mathcal{A}_{2}\right)}$ we have that $\mathbf{I}_{m_{1}}\left(\mathbf{1}_{\left\{f_{1} \geq t\right\}}\right) \geq \mathbf{I}_{m_{2}}\left(\mathbf{1}_{\left\{f_{2} \geq t\right\}}\right)$ for all $t \in[0,1]$. This means, by (I2*), $m_{1}\left(\left\{f_{1} \geq t\right\}\right) \geq m_{2}\left(\left\{f_{2} \geq t\right\}\right)$ for all $t \in[0,1]$, i.e. $h^{\left(m_{1}, f_{1}\right)}(t) \geq h^{\left(m_{2}, f_{2}\right)}(t)$ for all $t \in[0,1]$ and being $h^{(m, f)}:[0,1] \rightarrow[0,1]$ the function defined by $h^{(m, f)}(t)=m(\{f \geq t\})$ for all $t \in[0,1]$ and for any $(m, f) \in$ $\mathcal{D}_{[0,1]}$. By Theorem 1 since $\mathbf{I}$ is a universal integral, there exists a real valued nondecreasing function $\mathbf{J}$ such that $\mathbf{J}\left(h^{(m, f)}\right)=\mathbf{I}(m, f)$ for all $(m, f) \in \mathcal{D}_{[0,1]}$ (see also [23]). Being $h^{\left(m_{1}, f_{1}\right)} \geq h^{\left(m_{2}, f_{2}\right)}$ it follows that $\mathbf{J}\left(h^{\left(m_{1}, f_{1}\right)}\right)=\mathbf{I}_{m_{1}}\left(f_{1}\right) \geq$ 
$\mathbf{J}\left(h^{\left(m_{2}, f_{2}\right)}\right)=\mathbf{I}_{m_{2}}\left(f_{2}\right)$ and this proves (A1).

Axiom (A2) follows by (I2*). Indeed $\mathbf{I}\left(m, c \cdot \mathbf{1}_{X}\right)=c \otimes m(X)=c \otimes 1=c$, for all $c \in[0,1],(X, \mathcal{A}) \in \mathcal{S}$ and $m \in \mathcal{M}_{1}^{(X, \mathcal{A})}$.

Finally, (A3) is trivial, since for all $m \in \bigcup_{(X, \mathcal{A}) \in \mathcal{S}} \mathcal{M}_{1}^{(X, \mathcal{A})}$ we get $\mathbf{I}\left(m, \mathbf{1}_{E}\right)=$ $m(E)$ for all $E \in \mathcal{A}$ by $(\mathrm{I} 2 *)$.

4.1. Examples: developing Choquet and Sugeno integrals as families of aggregation functions satisfying axioms (A1)-(A3)

For all $(X, \mathcal{A}) \in \mathcal{S}$ and for all $m \in M_{1}^{(X, \mathcal{A})}$ let us consider the aggregation function $\boldsymbol{C}_{m}: \mathcal{F}_{[0,1]}^{(X, \mathcal{A})} \rightarrow[0,1]$ defined for all $f \in \mathcal{F}_{[0,1]}^{(X, \mathcal{A})}$ as

$$
C_{m}(f)=\int_{0}^{1} m(\{f \geq t\}) d t .
$$

If we consider the family $\mathcal{F}=\left\{\boldsymbol{C}_{m} \mid m \in \bigcup_{(X, \mathcal{A}) \in \mathcal{S}} M_{1}^{(X, \mathcal{A})}\right\}$, this satisfies axioms (A1)-(A3).

By Theorem 2 there exists a universal integral $\mathbf{C}: \mathcal{D}_{[0,1]} \rightarrow[0,1]$ with a semicopula $\otimes$ such that, for all $\mathbf{C}_{m} \in \mathcal{F} \cap \mathcal{H}^{(X, \mathcal{A})}$,

$$
\mathbf{C}_{m}(h)=\mathbf{C}\left(m_{\mathbf{C}_{m}}, h\right), \quad \text { for all } h \in \mathcal{F}_{[0,1]}^{(X, \mathcal{A})} .
$$

In this case the semicopula $\otimes$ is defined for all $(a, b) \in[0,1]^{2}$ by

$$
a \otimes b=\mathbf{C}_{m}\left(a \cdot \mathbf{1}_{B}\right)=\int_{0}^{1} m\left(\left\{a \cdot \mathbf{1}_{B} \geq t\right\}\right) d t=a \cdot m(B),
$$

with $\mathbf{C}_{m} \in \mathcal{F} \cap \mathcal{H}^{(X, \mathcal{A})}, B \in \mathcal{A}$ such that $\mathbf{C}_{m}\left(\mathbf{1}_{B}\right)=b$. By definition of $\mathbf{C}_{m}$, it follows that $m_{\mathbf{C}_{m}}=m$ and then

$$
a \otimes b=a \cdot m(B)=a \cdot m_{\mathbf{C}_{m}}(B)=a \cdot \mathbf{C}_{m}\left(\mathbf{1}_{B}\right)=a \cdot b .
$$

Thus, in this case the semicopula $\otimes$ is the standard product and, obviously, the universal integral $\mathbf{C}: \mathcal{D}_{[0,1]} \rightarrow[0,1]$ is the Choquet integral.

For all $(X, \mathcal{A}) \in \mathcal{S}$ and $m \in M_{1}^{(X, \mathcal{A})}$ let us consider the aggregation function $\boldsymbol{S}_{m}: \mathcal{F}_{[0,1]}^{(X, \mathcal{A})} \rightarrow[0,1]$ defined for all $f \in \mathcal{F}_{[0,1]}^{(X, \mathcal{A})}$ by $(\wedge$ indicates the minimum $)$

$$
\boldsymbol{S}_{m}(f)=\sup \{t \wedge m(\{f \geq t\}, t \in[0,1]\} .
$$


If we consider the family $\mathcal{F}=\left\{\boldsymbol{S}_{m} \mid m \in \bigcup_{(X, \mathcal{A}) \in \mathcal{S}} M_{1}^{(X, \mathcal{A})}\right\}$, this satisfies axioms (A1)-(A3).

By Theorem 2 there exists a universal integral $\mathbf{S}: \mathcal{D}_{[0,1]} \rightarrow[0,1]$ with a semicopula $\otimes$ such that, for all $\mathbf{S}_{m} \in \mathcal{F} \cap \mathcal{H}^{(X, \mathcal{A})}$,

$$
\mathbf{S}_{m}(h)=\mathbf{S}\left(m_{\mathbf{S}_{m}}, h\right), \quad \text { for all } h \in \mathcal{F}_{[0,1]}^{(X, \mathcal{A})} .
$$

In this case the semicopula $\otimes$ is defined for all $(a, b) \in[0,1]^{2}$ by

$$
a \otimes b=\mathbf{S}_{m}\left(a \cdot \mathbf{1}_{B}\right)=\sup \{t \wedge m(B), t \in[0, a]\}=a \wedge m(B),
$$

with $\mathbf{S}_{m} \in \mathcal{F} \cap \mathcal{H}^{(X, \mathcal{A})}, B \in \mathcal{A}$ such that $\mathbf{S}_{m}\left(\mathbf{1}_{B}\right)=b$. By definition of $\mathbf{S}_{m}$, it follows that $m_{\mathbf{S}_{m}}=m$ and then

$$
a \otimes b=a \wedge m(B)=a \wedge m_{\mathbf{S}_{m}}(B)=a \wedge \mathbf{S}_{m}\left(\mathbf{1}_{B}\right)=a \wedge b .
$$

Thus, in this second example the semicopula $\otimes$ is the minimum $\wedge$ and the universal integral $\mathbf{S}: \mathcal{D}_{[0,1]} \rightarrow[0,1]$ is the Sugeno integral.

\section{A second characterization of universal integral on the scale $[0,1]$}

From Theorem 2 we can elicit a new characterization of the family of universal integrals on the scale $[0,1]$, in the standard setting provided in [17].

Theorem 3 A function $\boldsymbol{I}: \mathcal{D}_{[0,1]} \rightarrow[0,1]$ is a universal integral on the scale $[0,1]$ if and only if it satisfies the following axioms:

(H1) $\boldsymbol{I}\left(m, \mathbf{1}_{E}\right)=m(E)$, for all $(X, \mathcal{A}) \in \mathcal{S}, m \in \mathcal{M}_{1}^{(X, \mathcal{A})}, E \in \mathcal{A}$;

(H2) $\boldsymbol{I}\left(m, c \cdot \mathbf{1}_{X}\right)=c$, for all $(X, \mathcal{A}) \in \mathcal{S}, m \in \mathcal{M}_{1}^{(X, \mathcal{A})}$ and $c \in[0,1]$;

(H3) $I\left(m_{1}, f_{1}\right) \geq I\left(m_{2}, f_{2}\right)$, for all $\left(m_{1}, f_{1}\right),\left(m_{2}, f_{2}\right) \in \mathcal{D}_{[0,1]}$ such that

$$
\left.\left.m_{1}\left(\left\{f_{1} \geq t\right\}\right) \geq m_{2}\left(\left\{f_{2} \geq t\right\}\right) \text { for all } t \in\right] 0,1\right] .
$$

\section{Proof}


First, let us suppose that I satisfies axioms (H1)-(H3). For all $(X, \mathcal{A}) \in \mathcal{S}$ and $m \in \mathcal{M}_{1}^{(X, \mathcal{A})}$ we consider the function $\boldsymbol{I}_{m}: \mathcal{F}_{[0,1]}^{(X, \mathcal{A})} \rightarrow[0,1]$ defined by $\boldsymbol{I}_{m}(f)=$ $\boldsymbol{I}(m, f)$ for all $f \in \mathcal{F}_{[0,1]}^{(X, \mathcal{A})}$. Due to axioms (H1)-(H3), $\boldsymbol{I}_{m}$ is an aggregation function from $\mathcal{H}^{(X, \mathcal{A})}$. Indeed, it holds: $\boldsymbol{I}_{m}\left(\mathbf{1}_{\emptyset}\right)=\boldsymbol{I}\left(m, \mathbf{1}_{\emptyset}\right)=m(\emptyset)=0$ and $\boldsymbol{I}_{m}\left(\mathbf{1}_{X}\right)=\boldsymbol{I}\left(m, \mathbf{1}_{X}\right)=m(X)=1$ by $(\mathrm{H} 1)$; for any pair of measurable functions $f, g \in \mathcal{F}_{[0,1]}^{(X, \mathcal{A})}$, with $g \leq f, m(\{g \geq t\}) \leq m(\{f \geq t\})$ for all $\left.\left.t \in\right] 0,1\right]$ and then $\boldsymbol{I}_{m}(g) \leq \boldsymbol{I}_{m}(f)$ by $(\mathrm{H} 3)$. Note that $\boldsymbol{I}_{m}$, according with (1), induces the same capacity $m$ : it holds that, for any $E \in \mathcal{A}, \boldsymbol{I}_{m}\left(\mathbf{1}_{E}\right)=m(E)$ by (H1).

Now consider the family of aggregation functions

$$
\mathcal{F}_{\boldsymbol{I}}=\left\{\boldsymbol{I}_{m} \mid m \in \bigcup_{(X, \mathcal{A}) \in \mathcal{S}} \mathcal{M}_{1}^{(X, \mathcal{A})}\right\} \subseteq \mathcal{H}_{[0,1]} .
$$

Using axioms (H1)-(H3), it is easily checked that $\mathcal{F}_{\boldsymbol{I}}$ satisfies axioms (A1)-(A3) and this concludes the sufficiency part.

The reverse of the proof (necessity part) is again easily proved. Indeed if $\boldsymbol{I}$ is a universal integral on the scale $[0,1]$, then the family $\mathcal{F}_{\boldsymbol{I}}$ defined in (6) satisfies axioms (A1)-(A3) and, consequently, $\boldsymbol{I}$ satisfies axioms (H1)-(H3).

Let us note that if $\mathbf{I}: \mathcal{D}_{[0,1]} \rightarrow[0,1]$ satisfies axioms $(\mathrm{H} 1)-(\mathrm{H} 3)$ then $\mathbf{I}$ is a universal integral on the scale $[0,1]$ (by Theorem 3 ) related to the semicopula $\otimes$ defined by $a \otimes b=\mathbf{I}\left(m, a \cdot \mathbf{1}_{E}\right)$ for all $a, b \in[0,1]$ and for some $(X, \mathcal{A}) \in \mathcal{S}$, $m \in \mathcal{M}_{1}^{(X, \mathcal{A})}$ and $E \in \mathcal{A}$ such that $m(E)=b$.

\subsection{An illustrative example}

Let us give an example of the use of Theorem 3. Consider the Choquet integral $\mathbf{C}: \mathcal{D}_{[0,1]} \rightarrow[0,1], \boldsymbol{C}(m, f)=\int_{0}^{1} m(\{f \geq t\}) d t$ and the Sugeno integral $\mathbf{S}:$ $\mathcal{D}_{[0,1]} \rightarrow[0,1], \boldsymbol{S}(m, f)=\sup \{t \wedge m(\{f \geq t\}, t \in[0,1]\}$. These are universal integrals on the scale $[0,1]$ related, respectively, to semicopulas product and minimum. Suppose we want to verify that the mixture $\alpha \mathbf{C}+(1-\alpha) \mathbf{S}: \mathcal{D}_{[0,1]} \rightarrow[0,1]$, defined by $(\alpha \mathbf{C}+(1-\alpha) \mathbf{S})(m, f)=\alpha \mathbf{C}(m, f)+(1-\alpha) \mathbf{S}(m, f)$, for $\alpha \in] 0,1[$, is again a universal integral on the scale $[0,1]$. In this case, it is sufficient (and trivial) to prove that $\alpha \mathbf{C}+(1-\alpha) \mathbf{S}$ satisfies axioms (H1)-(H3). Obviously, the underlying semicopulas is the mixture $a \otimes b=\alpha(a \cdot b)+(1-\alpha)(a \wedge b)$. 


\section{Conclusions}

The concept of universal integral generalizes several well known integrals used in Decision Analysis, like the Choquet integral and the Sugeno integral. In this paper we have provided two new axiomatizations of universal integrals on the scale $[0,1]$. The first one is in terms of a family of aggregation functions satisfying a set of desired properties. The second one is in the original setting of a functional defined on the union of all Cartesian products of the set of measures per the set of measurable functions. These new axiomatizations could represent a starting point for new theoretical and practical applications of the universal integral, both in MCDA as well as in decision under risk and uncertainty.

\section{Acknowledgment}

The work on this contribution was partially supported by the grants VEGA 1/0184/12 and GACR P 402/11/0378.

\section{References}

[1] M. Allais. Le comportement de l'homme rationnel devant le risque: Critique des postulats et axiomes de l'école Américaine. Econometrica, 21(4):503546, 1953.

[2] B. Bassan and F. Spizzichino. Relations among univariate aging, bivariate aging and dependence for exchangeable lifetimes. Journal of Multivariate Analysis, 93(2):313-339, 2005.

[3] S. Bodjanova and M. Kalina. Sugeno and Shilkret integrals, and T- and S-evaluators. In SISY 2009 - 7th International Symposium on Intelligent Systems and Informatics, pages 109-114, 2009.

[4] G. Choquet. Theory of Capacities. Annales de l'Institute Fourier (Grenoble), 5:131-295, 1953/54.

[5] D. Ellsberg. Risk, ambiguity, and the Savage axioms. The Quarterly Journal of Economics, 75(4):643-669, 1961.

[6] J. Figueira, S. Greco and M. Ehrgott. Multiple criteria decision analysis: state of the art surveys. J.R. Figueira, S. Greco, and M. Ehrgott, editors, Springer, Berlin, 2005. 
[7] I. Gilboa. Expected utility with purely subjective non-additive probabilities. Journal of Mathematical Economics, 16(1):65-88, 1987.

[8] M. Grabisch. The application of fuzzy integrals in multicriteria decision making. European Journal of Operational Research, 89(3):445-456, 1996.

[9] M. Grabisch and C. Labreuche. Bi-capacities-II: the Choquet integral. Fuzzy Sets and Systems, 151(2):237-259, 2005.

[10] M. Grabisch and C. Labreuche. Fuzzy measures and integrals in MCDA. In Multiple criteria decision analysis: state of the art surveys, pages 563-604. Springer Verlag, 2005.

[11] M. Grabisch, J.L. Marichal, R. Mesiar, and E. Pap. Aggregation functions: means. Information Sciences, 181(1):1-22, 2011.

[12] S. Greco, B. Matarazzo, and S. Giove. The Choquet integral with respect to a level dependent capacity. Fuzzy Sets and Systems, 175(1):1-35, 2011.

[13] S. Greco and F. Rindone. The Bipolar Choquet Integral Representation. Theory and Decision, (to appear). (DOI) 10.1007/s11238-013-9390-3.

[14] D. Kahneman and A. Tversky. Prospect theory: An analysis of decision under risk. Econometrica, 47(2):263-291, 1979.

[15] E.P. Klement, A. Kolesárová, R. Mesiar, and A. Stupňanová. Universal Integrals Based on Level Dependent Capacities. In IFSA-EUSFLAT. Citeseer, 2009.

[16] E.P. Klement, A. Kolesárová, R. Mesiar, and A. Stupňanová. A generalization of universal integrals by means of level dependent capacities. Knowledge-Based Systems, 38:14-18, 2013.

[17] E.P. Klement, R. Mesiar, and E. Pap. A universal integral as common frame for Choquet and Sugeno integral. IEEE Transactions on Fuzzy Systems, 18(1):178-187, 2010.

[18] R. Mesiar, A. Mesiarová-Zemánková, and K. Ahmad. Level-dependent Sugeno integral. IEEE Transactions on Fuzzy Systems, 17(1):167-172, 2009 . 
[19] D. Schmeidler. Integral representation without additivity. Proceedings of the American Mathematical Society, 97(2):255-261, 1986.

[20] D. Schmeidler. Subjective probability and expected utility without additivity. Econometrica, 57(3):571-587, 1989.

[21] N. Shilkret. Maxitive measure and integration. In Indagationes Mathematicae (Proceedings), volume 74, pages 109-116. Elsevier, 1971.

[22] C. Starmer. Developments in non-expected utility theory: The hunt for a descriptive theory of choice under risk. Journal of economic literature, 38(2):332-382, 2000.

[23] P. Struk. Extremal fuzzy integrals. Soft Comput., 10(6):502-505, 2006.

[24] M. Sugeno. Theory of fuzzy integrals and its applications. Ph.D. Thesis, Tokyo Institute of Technology, 1974.

[25] A. Tversky and D. Kahneman. The framing of decisions and the psychology of choice. Science, 211(4481):453, 1981.

[26] A. Tversky and D. Kahneman. Advances in prospect theory: Cumulative representation of uncertainty. Journal of Risk and uncertainty, 5(4):297323, 1992.

[27] J. Von Neumann, O. Morgenstern. Theory of games and economic behavior. Princeton university press, Princeton, NJ, 1947. 